\title{
Differential sensitivity of motor and sensory fibres in human ulnar nerve
}

\author{
J. L. VEALE ${ }^{1}$, R. F. MARK, AND SANDRA REES \\ From the Van Cleef Foundation Laboratory, Alfred Hospital and \\ Department of Physiology, Monash University, Melbourne, Australia
}

SUMMARY The human ulnar nerve has been stimulated with square-wave pulses of various fixed durations. Measurements were made of the growth of hand myograms compared with elbow neurograms and of hand myograms compared with finger neurograms, for fixed durations of pulses and increasing strength. The effect of blocking sensory action potentials with stimuli of various durations has been investigated, as well as the blocking of action potentials in motor nerve fibres by voluntary activity. It is concluded that pulses of long duration (1 msec or more) selectively stimulate sensory fibres at threshold, whereas short duration pulses (less than $200 \mu \mathrm{sec}$ ) selectively stimulate motor fibres.

It has been known for some years that sensory and motor nerve fibres have different electrical excitabilities. Stimulation of nerve trunks in experimental animals (Erlanger and Blair, 1938; Skoglund, 1942), showed that sensory and motor fibres differ in their responses to prolonged or slowly rising currents: sensory fibres accommodate less than motor fibres. This also holds for the stimulation of human nerves through the skin (Kugelberg, 1944). For current pulses of $1 \mathrm{msec}$ duration, Erlanger and Blair (1938) have shown that the rheobase of sensory fibres in the sciatic nerve of a bull frog is lower than that of motor fibres, but that with shorter currents the strength-duration curves cross and motor fibres become the more excitable. In man, Paillard (1955) plotted strength-duration curves for the monosynaptic $\mathrm{H}$ reflex and the direct motor response and found that the curves crossed in the same way. This suggested that for percutaneous stimulation of the posterior tibial nerve, the relative threshold of sensory and motor fibres reversed for short current pulses. Dawson (1956) investigated the sensitivity of motor and sensory fibres in the human ulnar and median nerves to stimulation by capacitor discharges and was able to stimulate selectively sensory fibres at threshold voltages. Buchthal and Rosenfalck
(1966) have reported extensive investigations into the sensitivity of sensory fibres in man, but largely confined their investigations to the use of pulses of $100 \mu \mathrm{sec}$ duration and mention no systematic study of the relative sensitivities of motor and sensory fibres.

Since excitability depends upon the duration of the current as well as on the intensity, we have investigated the sensitivity of sensory and motor fibres in human ulnar nerve to stimulation with square wave electrical pulses of varying durations and strength. The ulnar nerve was chosen as it has several regions where it may be stimulated or its activity recorded with comparative ease. We show that when short pulses are used (200 $\mu \mathrm{sec}$ or less) motor fibres are the more readily excitable, whereas, with long pulses (1 msec or more) sensory fibres are the more readily excitable.

\section{METHODS}

The subjects were seven normal adults, aged 20-40 years. All stimulation and recording were made through the skin. In order to reduce resistance at the electrodes the relevant skin areas were swabbed with chloroform, washed, and then gently abraded by light scrubbing with sandsoap or stroking with sterile needles. The areas were then re-washed and dried. 
The ulnar nerve was stimulated at the wrist using gauze-covered silver electrodes of the type described by Dawson and Scott (1949). The electrodes were placed just proximal to the wrist joint on the ventral surface of the forearm at the medial border. For stimulation of the digital branches of the ulnar nerve two narrow strips of platinum covered with gauze, were wrapped around the fourth and fifth fingers about $2 \mathrm{~cm}$ apart.

Ulnar neurograms were recorded from the elbow using silver electrodes placed over the medial epicondyle just proximal to the elbow joint. For recording ulnar neurograms from the fifth finger the electrodes described by Sears (1959) and McLeod (1966) were used: two 18 gauge platinum wires were wrapped around the finger. The myograms were recorded with small metal disc electrodes applied to the palmar surface of the hand over the hypothenar muscles. All electrode surfaces were smeared with Cambridge electrode jelly.
The potentials were recorded differentially and amplified by conventional AC coupled amplifiers with a band width of $2 \mathrm{~Hz}$ or less to $5 \mathrm{KHz}$ and displayed on a 565 Tektronix oscilloscope. A Devices Digitimer controlled the Mark IV Digitimer stimulators, one of which was modified to produce square wave pulses down to $5 \mu \mathrm{sec}$ duration, with a rise time of $1 \mu \mathrm{sec}$. Pulse durations employed ranged from $5 \mu \mathrm{sec}$ to $2 \mathrm{msec}$ but were usually between $50 \mu \mathrm{sec}$ and $1 \mathrm{msec}$. The output impedance of this stimulator was checked and found to be $230 \Omega$, well within the manufacturer's specification.

Averaged responses were obtained using a Mnemotron Computer of Average Transients (CAT) Model 400B, whose action was modified in the following manner. Trains of pulses were generated which advanced the CAT part way through its memory stores (using the External Address Advance input). In this way the CAT could be advanced through a single sweep in controllable short seg-

(A)

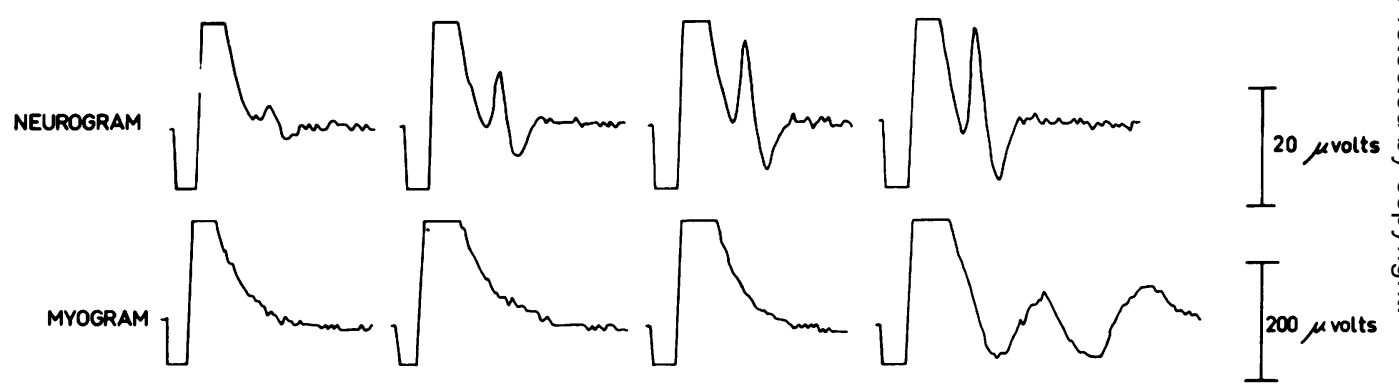

1 msec duration VOLTAGE:

4V

(B)
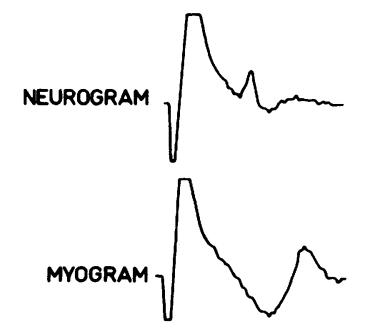

10 usec duration VOLTAGE:
$82 \mathrm{~V}$

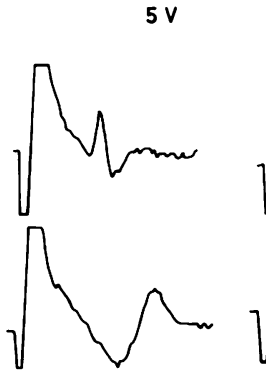

$84 \mathrm{~V}$
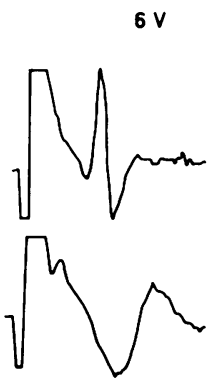

$88 \mathrm{~V}$.
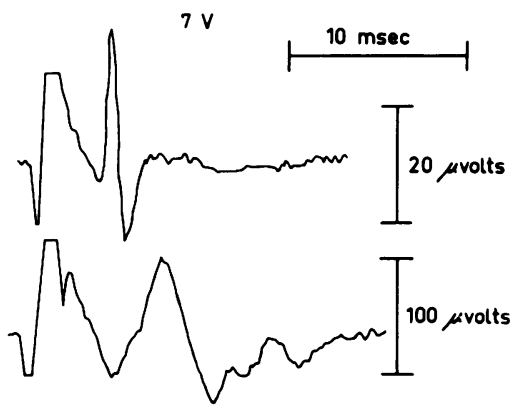

$90 \mathrm{~V}$

FIG. 1. (A) Four simultaneous pairs of recordings of ulnar neurograms recorded at the elbow (upper records) and hand myograms (lower records) obtained using pulses of 1 msec duration and voltages as indicated under the myograms. Each record is an average of 50 sweeps, using the CAT. The time scale applies to all records, the vertical scales apply to the records in their row. (B) The same, with pulses of $10 \mu \mathrm{sec}$ duration. The growth of a neurogram before the appearance of a myogram with a pulse duration of $1 \mathrm{msec}$ and increasing strength of stimulus is seen in records (A), whereas (B) shows similar growth of both neurogram and myogram with increasing strength when the pulse duration is $10 \mu \mathrm{sec}$. 
ments. Thus two (or more) different stimulating procedures could be used sequentially, and the responses to each procedure stored in consecutive segments of the CAT sweep. This whole sequence could then be repeated to obtain averaging of the different responses. In this way there could be controllable pauses between the segments, with only the portions of the responses of interest being averaged and the time resolution of the averaging period controllable. Any slow change in the recording situation, such as drying out of electrodes, could be expected to affect the averaged responses in all segments equally. Thus more reliable results were obtained than if averages for the first procedure had been obtained first, and then averages for the second, and so on.

The output from the CAT was written out on a Moseley X-Y plotter. Whenever reference is made to the amplitude of a neurogram or myogram it implies the result of averaging between 20 and 50 repetitions of the stimuli. The height of the stimulus artefact, displayed on the X-Y plotter does not have any simple quantitative relationship with stimulus strength. The recorded artefact exceeded the permissible linear input range of the CAT, and the Vertical Range setting selected for plotting the memory content generally resulted in memory overflow during the artefact.

We have used the assumption that within a restricted range of fibre diameters the amplitude of the neurogram will reflect the number of fibres stimulated.
When current flow through the tissues was measured, two methods were used: (1) an $0.1 \mathrm{~K} \Omega$ resistor was inserted in series with the stimulating elctrodes, and the voltage drop developed across it recorded. In this case amplification with a bandwidth from D.C. to $75 \mathrm{kHz}$ was used. (2) A Tektronix P6016 current probe with type 131 amplifier was used. This had a rise time of below a microsecond, and an adequate steady state response for the current durations employed.

\section{RESULTS}

\section{Four types of experiment were performed:}

DIFFERENTIAL GROWTH OF NEUROGRAMS AND MYOGRAMS The ulnar nerve was stimulated at the wrist, and simultaneous recordings made of the resultant neurogram at the elbow and the myogram in the hand. The myogram had a constant latency of only a few msec, and was thus a result of direct stimulation of motor fibres and not reflex in origin. With various fixed pulse durations, the strength of the stimulus was increased from threshold voltage upwards in 1-3 V steps. At each voltage, averages of 20-50 responses were obtained and the amplitudes of the neurogram and myogram measured.

Figure 1 shows a series of records taken from one subject. With a pulse duration of $1 \mathrm{msec}$ a

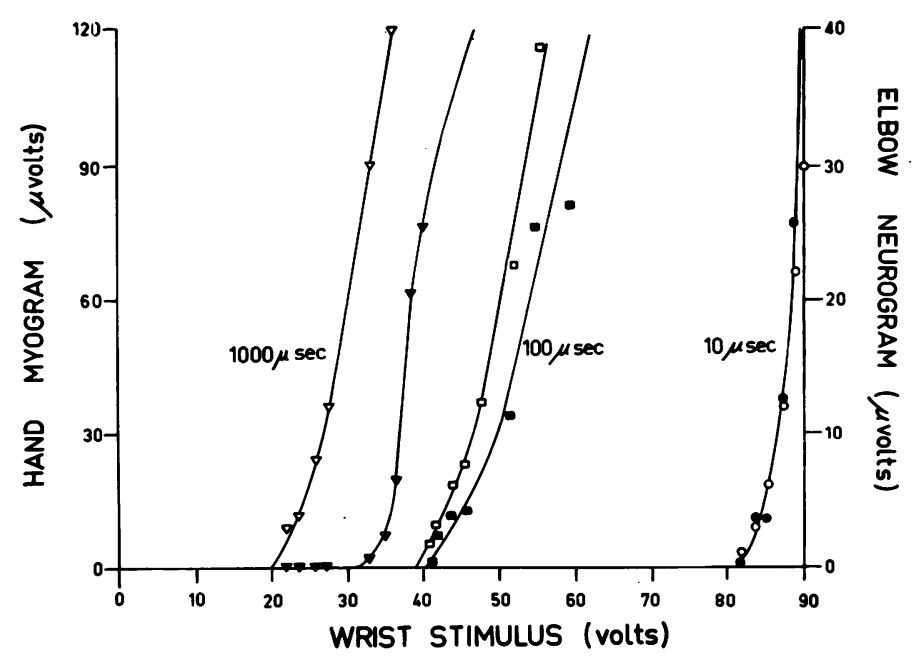

FIG. 2. Graph of the amplitudes of the hand myograms and elbow neurograms plotted against voltage of the stimulus applied at the wrist, for three durations of stimulus as marked (10 $\mu \mathrm{sec}, 100 \mu \mathrm{sec}$, and 1,000 $\mu \mathrm{sec}$ ). Open symbols for neurograms, filled for myograms. $\nabla: 1,000$ usec;

ㅁ: $100 \mu \mathrm{sec} ; \bigcirc: 10 \mu \mathrm{sec}$. The left hand scale applies to the myograms, the right hand to the neurograms. The relative size of these scales has been chosen so that the points for myograms and neurograms elicited by a pulse duration of $10 \mu \mathrm{sec}$ lie on one curve. The existence of a neurogram with no myogram for a pulse duration of 1,000 $\mu \mathrm{sec}$ is clearly seen, as well as some separation of the curves with 100 $\mu \mathrm{sec}$ pulse durations. 


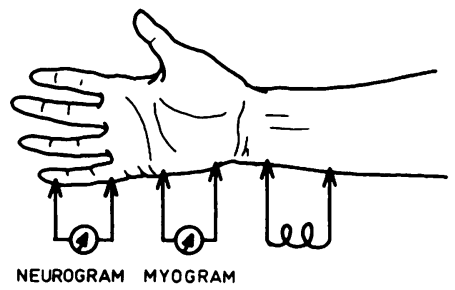

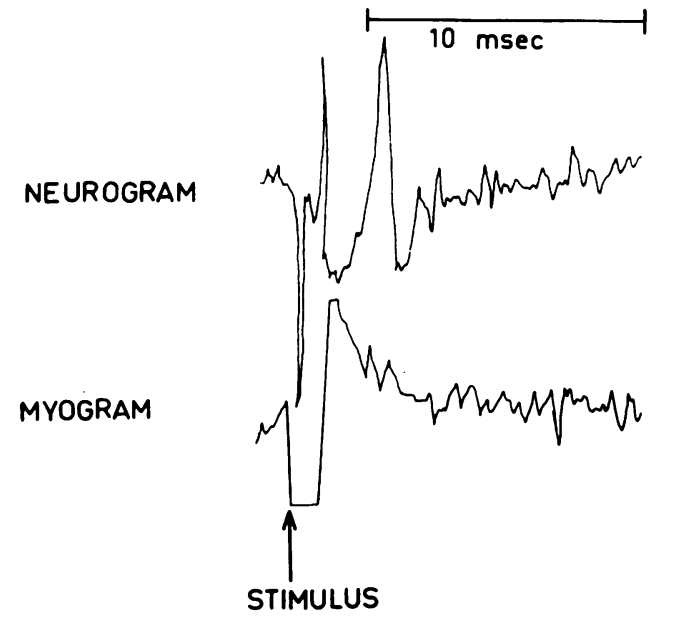

PULSE SIZE::

$1 \mathrm{msec}, 35$ volts

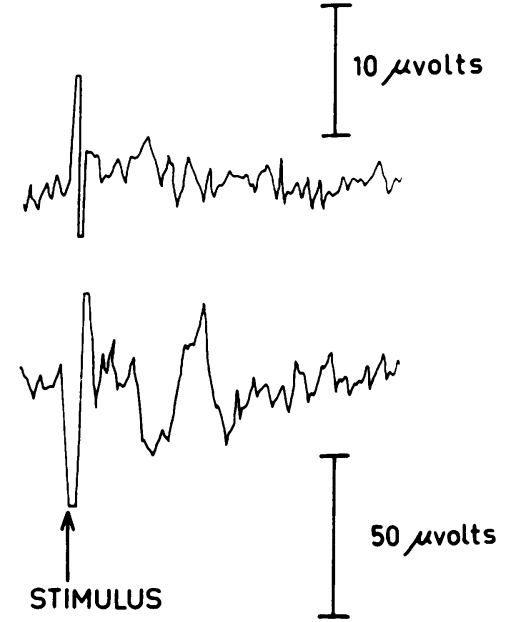

$50 \mu \mathrm{sec}, 65.5$ volts

FIG. 3. A diagram of the electrode arrangements for stimulating the ulnar nerve at the wrist and recording the hand myogram and finger neurogram. Two sets of averages of a finger neurogram (upper row) obtained simultaneously with a hand myogram (lower row) with two pulse durations and strengths as marked under each (simultaneous) pair. The time scale applies to all records, the vertical scale to recordings of the same row. The stimulus was applied at the time indicated by the arrow. The averages are a result of 50 repetitions, with the two pulses being applied alternately at 500 msec intervals, and the ensuing responses stored appropriately in one of two separate segments of the CAT memory. At $1 \mathrm{msec}$ pulse duration, there is a neurogram but no myogram; at 50 usec pulse duration a myogram but no neurogram.

neurogram was obtained at threshold without any recordable myogram. As the stimulus strength was increased a myogram appeared. With a pulse duration of $10 \mu \mathrm{sec}$ both a neurogram and myogram were recorded at threshold and they grew together as the stimulus strength was increased.

Curves of the growth in amplitude of the neurogram and myogram were plotted for each fixed pulse duration. Figure 2 shows curves plotted for pulse durations of 10,100 , and 1,000 $\mu \mathrm{sec}$. For the sake of clarity other pulse durations have not been plotted in the figure. The curves of the neurograms and myograms pro- duced by $10 \mu \mathrm{sec}$ pulses grew at exactly the same rate and the vertical scales were chosen so that the points for this pulse duration fell along one curve. With $100 \mu \mathrm{sec}$ pulses the voltage thresholds for both responses were reduced but that for the neurogram was significantly lower. At $1,000 \mu \mathrm{sec}$ duration a large neurogram could be elicited with stimulus voltages that gave no myogram at all.

This experiment was carried out in six of the subjects, and in all cases gave results comparable with those described. Not every experiment gave such a degree of separation of the responses at longer durations as illustrated in Fig. 2, but in 


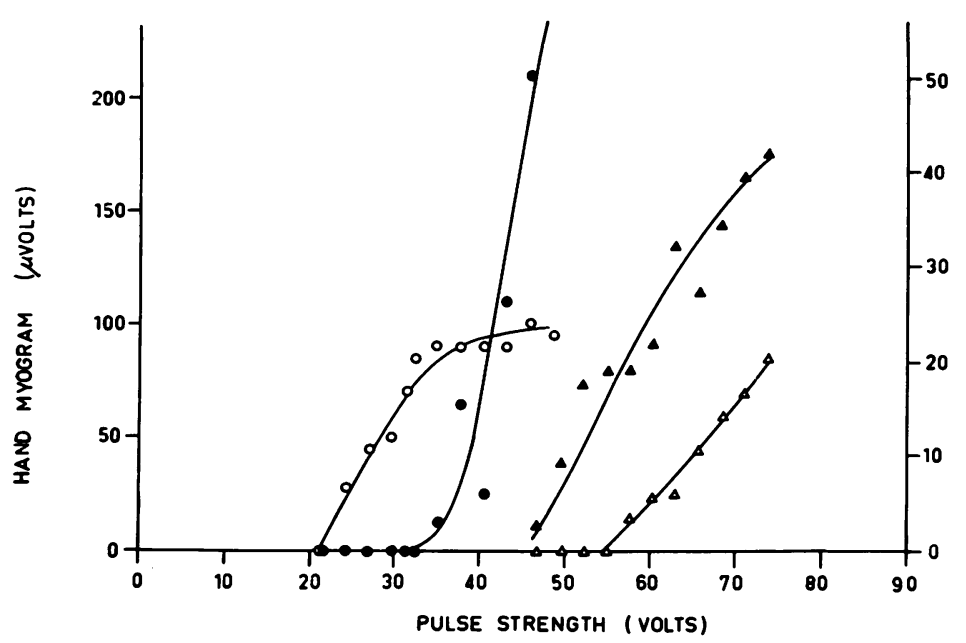

FIG. 4. Amplitudes of hand myograms (left hand scale) and finger neurograms (right hand scale) after wrist stimulation with fixed pulse duration, plotted against stimulus strength. Filled symbols: myograms. Open symbols: neurograms. $\bigcirc$ and O: 1 msec pulse duration. $\triangle$ and $\triangle: 50 \mu$ sec pulse duration. The curves show clearly that, with increasing strength of stimulation there is initially growth of the neurogram with no myogram at 1 msec duration, and growth of the myogram with no neurogram at $50 \mu \mathrm{sec}$ duration. all cases a similar dependence of threshold on pulse duration could be demonstrated.

RECORDING OF DIGITAL NEUROGRAMS AND HAND MYOGRAMS The ulnar nerve was stimulated at the wrist and simultaneous recordings made of the myogram in the hand, and the neurogram in the fifth finger. Because of the lack of any motor innervation in the finger, this neurogram is entirely sensory. With selected pulse durations, the growth in neurogram and myogram amplitudes with increasing strength of stimuli were measured.

The sweep of the CAT was split into two segments. Pulses of different duration and amplitude were used in each segment and the separate responses averaged. Figure 3 shows the result of one such experiment, with the strengths adjusted to be just above threshold for either a sensory or motor response. It is clear that, with a pulse duration of $1 \mathrm{msec}$, there is a sensory neurogram with no motor activity indicated in the myogram, whereas for a pulse duration of $50 \mu \mathrm{sec}$ there is a recordable myogram with no neurogram.

The overall results for such an experiment are shown in Fig. 4. The differential sensitivity of the motor and sensory fibres to pulse duration can be easily seen. A similar result was obtained in a second subject.

It must be pointed out that this type of result is obtained only after careful positioning of the recording electrodes. If the electrodes are not favourably positioned it is possible to record either sensory or motor responses first at threshold for any of the stimulus durations used. Nevertheless, a difference in the relative sensitivities of motor and sensory fibres can always be demonstrated. For instance, if the electrode placement is such that the myogram appears at a lower strength of stimulation than the sensory neurogram, it is found that to elicit a neurogram, the increase in stimulus strength required (over and above the threshold for the myogram) is less with long pulse durations than with short.

COLLISION IN SENSORY FIBRES The digital branches of the ulnar nerve in the fourth and fifth fingers were stimulated and the strength adjusted to produce a significant neurogram at the elbow. Again, since the ulnar innervation of these fingers is entirely sensory, this neurogram must reflect activity in sensory fibres. The ulnar nerve was then stimulated at the wrist, with some selected pulse duration and the strength adjusted until the neurogram induced at the elbow was of comparable magnitude to the sensory neurogram obtained by finger stimulation.

The ulnar neurogram was then averaged with the CAT sweep split into three segments. The first segment averaged the ulnar neurogram at the elbow arising from finger stimulation alone. The second segment averaged the ulnar neuro- 

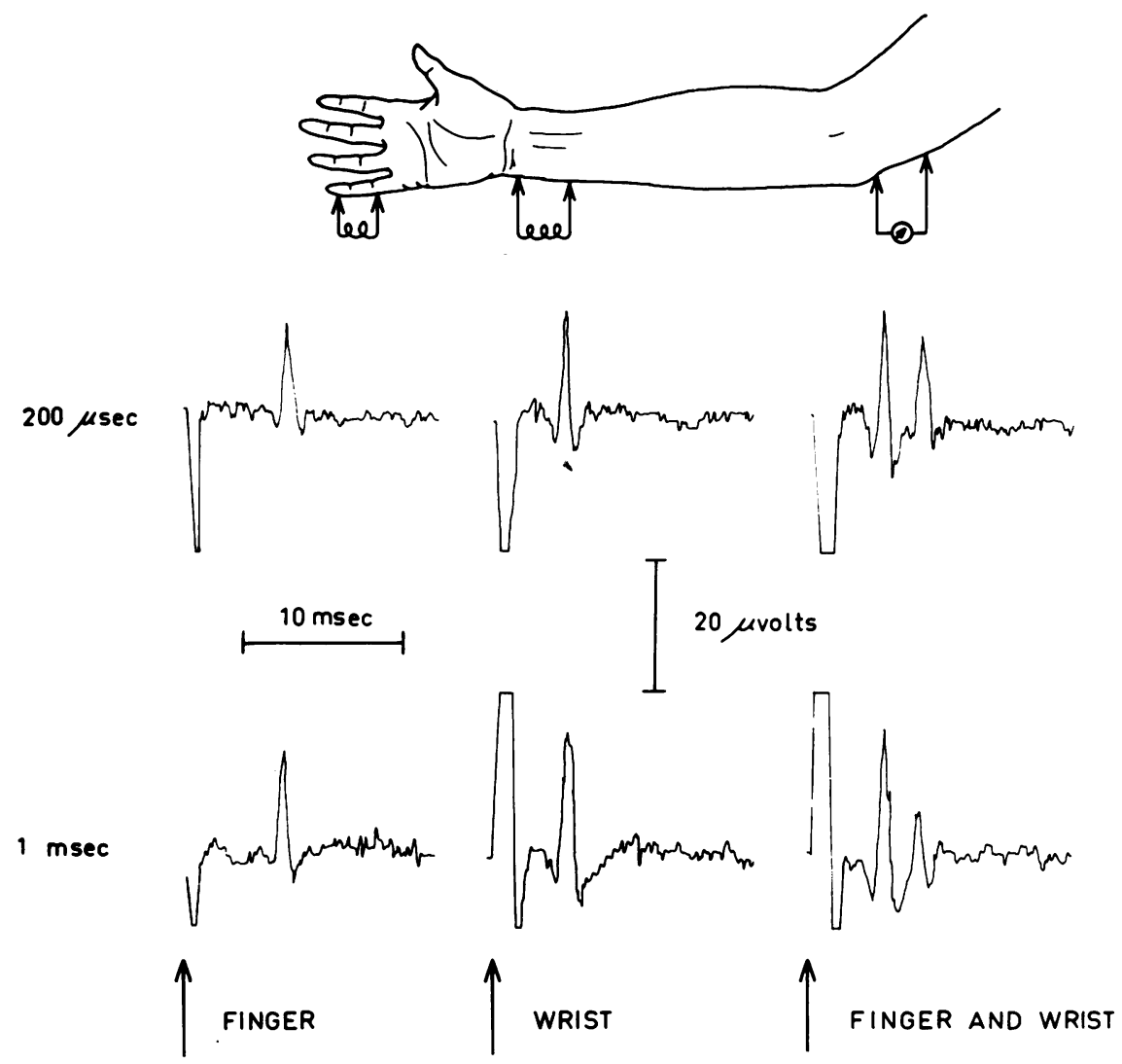

FIG. 5. Diagram of stimulating sites (wrist and finger) and recording site (elbow) for the experiments involving collision in sensory fibres. Graphs of two sets of records obtained for two different pulse durations as marked at the left of each set of three records. The time and voltage scales apply to all records. Each record is an average of the responses to 50 repetitions of the stimulus, which is applied at the time indicated by the arrow. Three different stimuli have been used, separated by 200 msec intervals, and the sequence repeated 50 times: (1) finger stimulation alone (left hand record), (2) wrist stimulation alone (middle record), (3) wrist and finger simultaneously (right hand record). The second peak in the third record is the sensory neurogram produced by finger stimulation, and has plainly been reduced by the simultaneous wrist stimulation when the wrist pulse is 1 msec in duration, and much less so when of $200 \mu \mathrm{sec}$ duration.

gram arising from wrist stimulation. The third segment averaged the ulnar neurogram arising from simultaneous stimulation of the fingers and wrist. The moment of stimulation in each segment coincided with the start of that segment. Two examples of the average results of $50 \mathrm{such}$ sweeps are shown in Fig. 5 for pulse durations of $200 \mu \mathrm{sec}$ and $1 \mathrm{msec}$.

Consider the amplitudes of the neurograms produced by finger stimulation in the third segment (the second peak) compared with that in the first segment. It is plain that at long durations of wrist pulses $(1 \mathrm{msec})$, there is a marked diminution in its height whereas for short pulses $(200 \mu \mathrm{sec})$ there is little change. Results such as these were obtained for intermediate pulse durations, and have been plotted in Fig. 6. The height of the neurogram arising from finger 


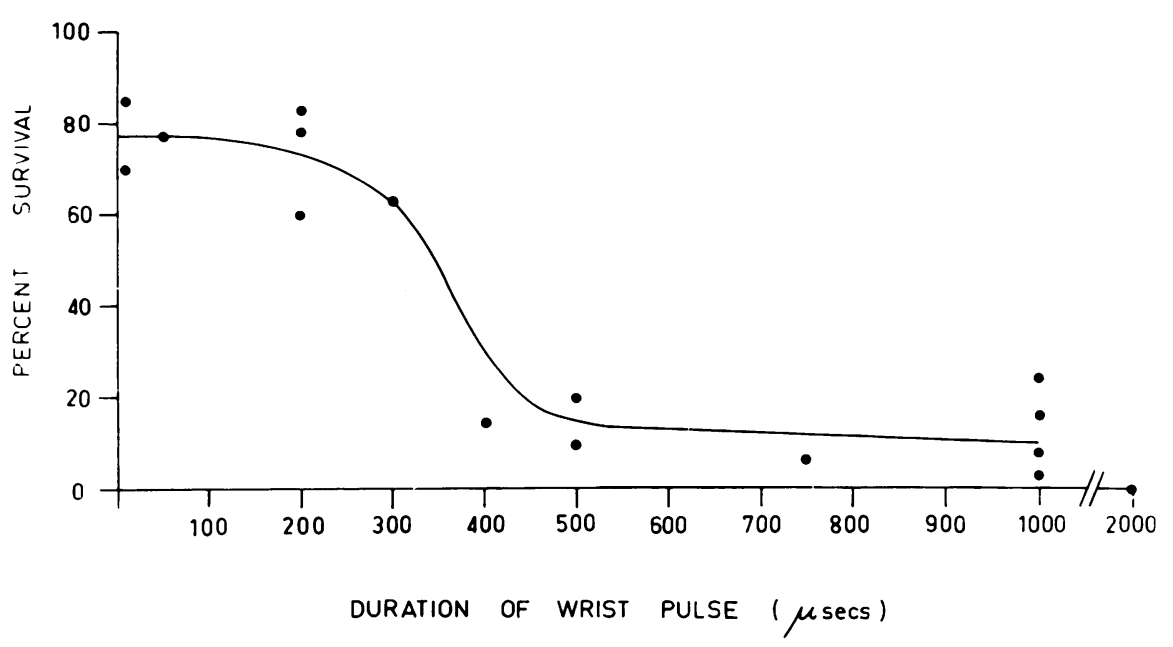

FIG. 6. The results of experiments as illustrated in Fig. 5. The height of the neurogram produced by finger stimulation with simultaneous wrist stimulation (second peak of third record in Fig. 5) is expressed as a percentage of the height of the neurogram produced by finger stimulation alone (peak of first record in Fig. 5) and has been called the percent survival. This is here plotted as a function of the duration of the pulse applied at the wrist. In each experiment the strength of the wrist stimulus was adjusted so that the neurogram produced by it alone was constant irrespective of pulse duration. The blocking effect of the wrist pulse at long durations is easily seen. In this case a pulse of 2 msec duration was needed to occlude the sensory neurogram completely.

stimulation with simultaneous wrist stimulation - that is, second peak, segment three-has been expressed as a percentage of the neurogram arising from finger stimulation alone-that is, the peak in segment one. This percentage has been called the percent survival of the sensory neurogram and is plotted as a function of the duration of the wrist pulse. It is plain from the graph that the percent survival is high for short pulse durations, low for long pulse durations, with a fairly abrupt changeover around 350 $\mu \mathrm{sec}$ in this particular instance. Presumably where a reduction in the amplitude of the neurogram has occurred, it indicates that proximally propagated sensory action potentials arising from finger stimulation (the digital branches of the ulnar nerve being entirely sensory) have collided with antidromically propagated action potentials arising from wrist stimulation. As a significant reduction occurred only with long duration pulses it indicates that such pulses selectively stimulate sensory fibres.

This type of experiment was done on five sub- jects and the results were similar in all cases. Pulses of long duration always resulted in a marked reduction in the height of the sensory neurogram, whereas pulses of short duration had little effect.

COLLISION IN MOTOR FIBRES With the arm relaxed, the ulnar nerve was stimulated at the wrist, and the evoked ulnar neurograms at the elbow averaged for 50 stimuli. The subject was then asked to contract those hand muscles which had been contracting during the previous stimulation. While this voluntary contraction was maintained, another 50 stimuli were applied to the wrist, and the evoked ulnar neurograms again averaged. A third recording with the hand relaxed was then made. If the third recorded neurogram was markedly different from the first (both taken in the relaxed state) then these results were discarded, it being presumed that the stimulating or recording electrodes had moved as a result of the voluntary contraction.

Examples of the average results to 50 stimuli 

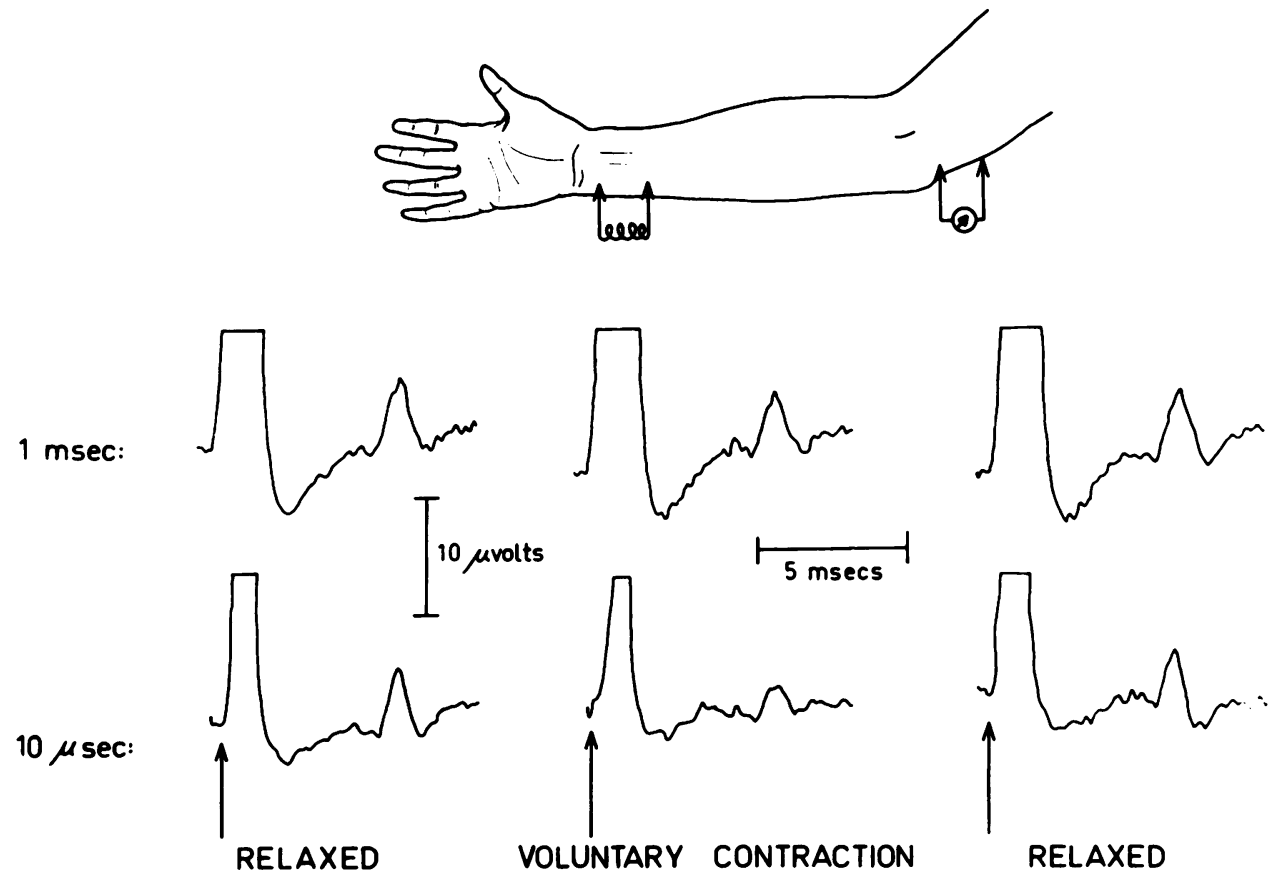

FIG. 7. Diagram of stimulating site at wrist and recording site at elbow. Records of ulnar neurograms obtained with stimulation at the wrist, each record being the result of averaging 50 responses. Upper row: pulse duration of $1 \mathrm{msec}$; lower row: $10 \mu \mathrm{sec}$. Left hand records: hand relaxed. Middle records: hand contracted. Right hand records: hand relaxed again. The stimulus is applied at the time indicated by the arrow. With contraction the neurogram produced by the $10 \mu \mathrm{sec}$ pulse has clearly been more reduced in amplitude than that produced by a 1 msec duration pulse.

for two different pulse durations are shown in Fig. 7. The height of the neurogram during voluntary contraction can be expressed as a percentage of the average neurogram in the relaxed states (before and after the contraction) yielding a percent survival figure. Figure 8 shows the percent survival expressed as a function of stimulus duration. The use of a semi-log plot is purely for diagrammatic clarity, and the dashed line has been inserted by eye. The six points for three pulse durations show that reasonably reproducible results could be obtained in this subject. This experiment was attempted in three subjects, and the results presented here from one subject were the most satisfactory.

It is clear that ulnar neurograms elicited by $10 \mu \mathrm{sec}$ pulses were reduced by voluntary contraction, whereas neurograms elicited by $1 \mathrm{msec}$ pulses were not. We interpreted this as indicating that antidromic action potentials generated in motor fibres by wrist pulses of $10 \mu \mathrm{sec}$ duration had collided with orthodromic action potentials resulting from the subject's voluntary effort. The lack of a significant reduction in the neurogram elicited by $1 \mathrm{msec}$ duration pulses indicated that sensory fibres rather than motor fibres were being stimulated.

CURRENT FLOW WITH CHANGING PULSE DURATIONS On three occasions the current flow during stimulation with various pulse durations was measured for stimulation of the ulnar nerve at the wrist. With $50 \mu \mathrm{sec}$ pulse durations a reasonably rectangular pulse of current flow occurred as shown in Fig. 9A. The applied voltage divided by the peak magnitude of current yielded an apparent resistance of $1 \cdot 3 \mathrm{~K} \Omega$. For $1,000 \mu \mathrm{sec}$ pulse durations there was initially a 


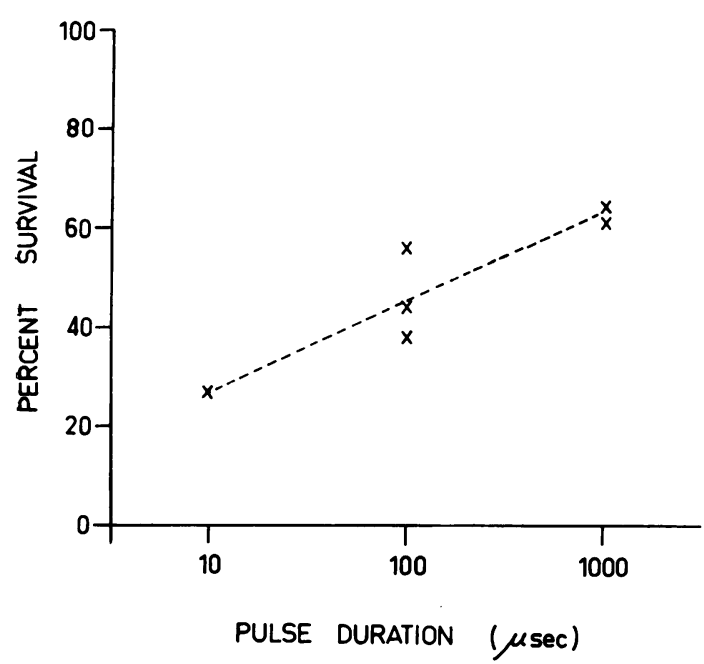

FIG. 8. Graph of percent survival plotted against pulse duration in a motor collision experiment, of the type shown in Fig. 7. The height of the neurograms following wrist stimulation during muscle contraction has been expressed as a percentage of the average neurograms obtained in the relaxed state before and after the contraction. This percentage has been called the percent survival. The use of a log scale for pulse duration is purely for diagrammatic clarity. The dashed line has been inserted by eye. The six values (indicated by crosses) were obtained during one experiment. The neurogram induced by short duration pulses is more readily blocked by voluntary contraction than that produced by long pulses.

$A$

B
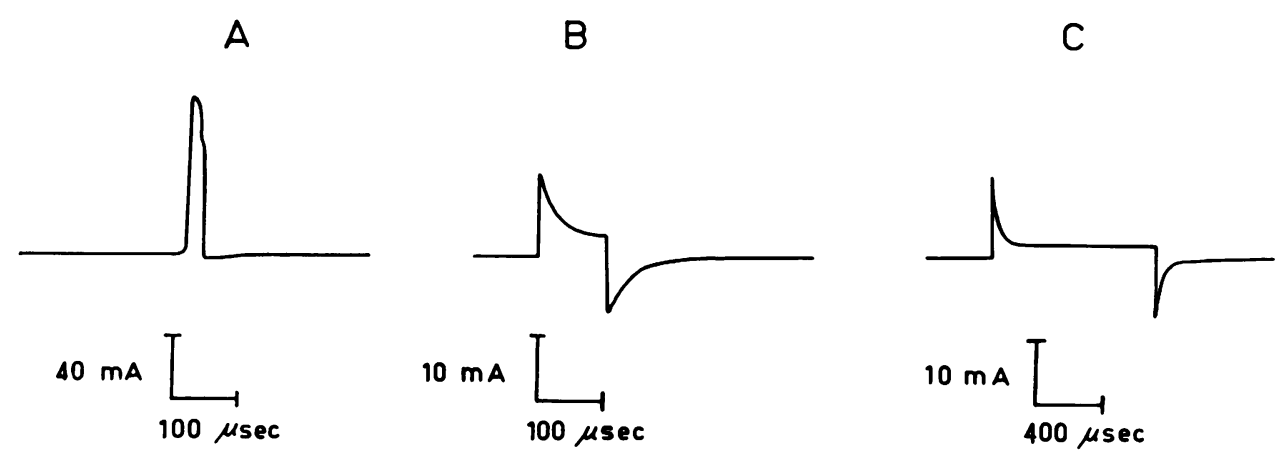

marked overshoot of current flow, with a rapid decay over about $100 \mu \mathrm{sec}$ to a plateau of steady current flow (Fig. 9B). At the cessation of the voltage stimulus there was a reversal of current flow, mirroring the overshoot, and a comparable rapid return to zero. The steady plateau of current flow was plotted as a function of applied voltage and yielded a good linear relationship indicating a resistance of $4 \mathrm{~K} \Omega$ in the tissues. The initial peak amplitude of current was also plotted and yielded a smooth curve, concave downwards, but always increasing with increasing strength of voltage. In magnitude this current was two to four times greater than the plateau value. These are plotted in Fig. 10.

With intermediate pulse durations there were

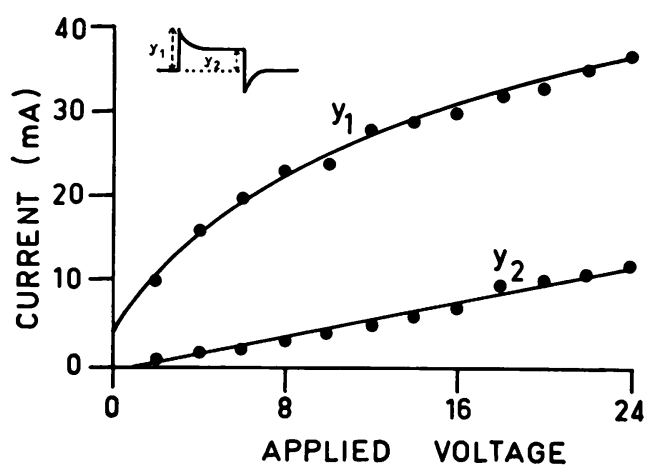

FIG. 10. Graphs of the current flow, with a $1 \mathrm{msec}$ duration of pulse, plotted against stimulus voltage. The graph $\mathrm{y}_{1}$ is the initial peak of current flow, and $\mathrm{y}_{2}$ the plateau level, as indicated in the sketch.

FIG. 9. Current waveforms for three durations of pulses: $25 \mu \mathrm{sec}$ (left), $100 \mu \mathrm{sec}$ (middle), 1 msec (right). 
comparable findings. At $100 \mu \mathrm{sec}$ pulse duration there was no period with a steady plateau of current, and at $500 \mu \mathrm{sec}$ duration there was a $400 \mu \mathrm{sec}$ plateau period. It is plain that there are different patterns of current flow with short and long pulse durations.

\section{DISCUSSION}

This paper reports the first attempt to investigate systematically the electrical threshold of sensory and motor fibres in human peripheral nerves to square wave electrical pulses of varying duration. Sensory fibres are more easily excited by longer pulses and motor fibres become more sensitive with brief pulses. The differences in properties are great enough in the human ulnar nerve to allow stimulation of either motor or sensory fibres near threshold with little or no contamination from the other group.

The above conclusion was based on four lines of evidence. When the ulnar nerve was stimulated at the wrist with long duration pulses (1 msec or more) the neurogram recorded at the elbow with threshold voltages was presumably indicating activity in sensory fibres as no myogram was recorded. Since the neurogram produced by short duration pulses ( $200 \mu \mathrm{sec}$ or less) grew exactly in proportion to the myogram as the stimulus strength was increased, it was presumably indicating activity in motor fibres. If either the myogram or neurogram were relatively insensitive indicators of activity in the nerve or muscle respectively, this argument would be weakened. One might have sensory impulses passing up the ulnar nerve or contraction of hypothenar muscles without recordable results.

The same objection could be applied to the second experiment in which simultaneous recordings were made of the digital neurograms and hand myograms resulting from wrist stimulation with pulses of varying duration. However the observation that one can record a neurogram at threshold but no myogram with a long duration pulse and a myogram at threshold but no neurogram with a short duration pulse, make it more likely that these results can be explained in terms of a differential sensitivity of motor and sensory fibres to the duration of the stimulating pulse.
The problem of the relative sensitivity of the two recording sites was overcome in the third experiment. Only one recording site was used and there is no reason to suppose that the ease of obtaining records from sensory or motor fibres at the same site differs. Long duration pulses delivered at the wrist were more effective than short duration pulses in blocking sensory action potentials generated by stimulation of the digital branches of the ulnar nerve. We feel that this experimental result, consistently obtained in five subjects, provides the strongest evidence that sensory and motor fibres have a differential sensitivity to pulse duration.

The sensory collision experiment is an extension of Dawson's (1956) experiment in which he showed that sensory fibres to the fingers have a lower electrical threshold than motor fibres to the small muscles of the hand. We point out that we have used square wave pulses of varying duration, whereas Dawson used capacitor discharges and does not report any results from systematic variation in the time constant.

In the motor collision experiment the chance्षे of collision was not high. The sustained rate of occurrence of action potentials in a single fibre during a contraction is of the order of 50/sece (Marsden, Meadows, and Merton, 1971). The velocity of conduction is, say, $55 \mathrm{~m} / \mathrm{sec}$, and the distance over which collision can occur and influence the neurogram is about $30 \mathrm{~cm}$. Calculation shows that a collision of action potentials would be predicted in about one half of the active fibres, and therefore a fall in the ulnar neurogram to about $50 \%$ of the value obtained during relaxation. Thus the effect cannot be expected to be great. This experiment was technically difficult to perform. Much depended upon the ability of the subject to contract his hand muscles strongly without disturbing the electrodes.

THEORETICAL ASPECTS If the relative electrical thresholds of motor and sensory fibres depends on the duration of the stimulating pulse, there could be some anatomical difference-for example, in diameters-between the fibres, combined with a difference in their membrane properties. McLeod and Wray (1967) showed in the baboon that the ratio of conduction velocity (in $\mathrm{m} / \mathrm{sec}$ ) to diameter (in $\mu$ ) is 4.1 for motor 
fibres and $5 \cdot 2$ for sensory. This presumably does reflect a difference in membrane properties. Furthermore, anatomically, the motor fibres are axons and the sensory fibres are dendrites, making it plausible that there could be systematic differences between them.

Threshold is dependent on the short time factor of excitation, $k$ (Hill, 1936a, b). This is a measure of the passive electrical properties of the nerve membrane and influences the shape of the strength-duration curve. With $k$ fixed the rheobase will depend on fibre diameter. If the diameters of two groups of fibres were similar but not identical and had different values of $k$, the curves could cross. For example, if motor fibres were only slightly smaller than sensory fibres but their membrane time constant shorter, then the motor fibres could have a lower threshold than sensory for short pulse durations, but a higher one for long durations. The differing rates of accommodation of sensory and motor fibres ( $\lambda$ of Hill, 1936b) presumably reflects differences in the rate of voltage-dependent inactivation of sodium permeability (Hodgkin, 1964). The time required is normally longer than that of the pulses used in these experiments.

Differences in physical parameters of nerve fibres must be very small and their magnitude could be worked out in animal preparations where the exact shape of the stimulating pulse could be measured and correlated with local potential and excitability changes in nerve fibres. The present experiments on man show that it is possible to stimulate at threshold either the sensory or motor fibres in the ulnar nerve by changing the pulse length. We consider that this is a useful new method in human neurophysiology and have already used it to demonstrate Renshaw cell activity in man (Veale, Rees, and Mark, 1972.

\section{SUMMARY}

Seven normal human subjects (aged 20-40 years) were investigated to assess the sensitivity of the ulnar nerve to stimulation with square wave pulses of varying duration and strength.

Four types of experiment were performed; in each experiment the ulnar nerve was stimulated at the wrist. (1) Simultaneous recording of the ulnar neurogram at the elbow and the hand myogram (of ulnar innervated muscles) showed that, for short duration pulses (less than 200 $\mu \mathrm{sec})$, with increasing strength of stimuli the amplitude of the myograms and neurograms increased together, whereas for long duration pulses (1 msec or more) a neurogram was recorded at the lower stimulus strengths with no myogram. (2) Simultaneous recording of a digital neurogram (sensory) and hand myogram (motor) showed that long duration pulses at threshold for a sensory neurogram produced no myogram, whereas short duration pulses at threshold for a myogram produced no neurogram. (3) Long duration pulses were more effective than short duration pulses in blocking sensory action potentials produced by stimulation of the digital branches of the ulnar nerve in the fourth and fifth fingers. (4) The neurograms generated in the ulnar nerve by short duration pulses were more effectively blocked during voluntary contraction of ulnar innervated muscles than were the neurograms generated by long duration pulses.

It was concluded that pulses of long duration (1 msec or more) stimulate sensory fibres at threshold, whereas short duration pulses (less than $200 \mu \mathrm{sec}$ ) stimulate motor fibres. A possible basis for this differential sensitivity of motor and sensory fibres is discussed.

We are grateful to Miss Louise Keegan for her assistance in the laboratory and for preparation of the illustrations. The work was done in the Van Cleef Laboratory and funded by the Van Cleef Foundation and grant (No. 69/4676) from the National Health and Medical Research Council of Australia. We thank Dr. Brian Cragg for his helpful advice and Professor R. Porter for reading the manuscript.

\section{REFERENCES}

Buchthal, F., and Rosenfalck, A. (1966). Evoked action potentials and conduction velocity in human sensory nerves. Brain Research, 3, 1-122.

Dawson, G. D. (1956). Relative excitability and conduction velocity of sensory and motor nerve fibres in man. Journal of Physiology, 131, 436-451.

Dawson, G. D., and Scott, J. W. (1949). Recording of nerve action potentials through skin in man. Journal of Neurology, Neurosurgery, and Psychiatry, 12, 259-267.

Erlanger, J., and Blair, E. A. (1938). Comparative observations on motor and sensory fibers with special reference to repetitiousness. American Journal of Physiology, 121, 431453. 
Hill, A. V. (1936a). Excitation and accommodation in nerve. Proceedings of the Royal Society $B, 119,305-355$.

Hill, A. V. (1936b). Strength-duration relation for electric excitation of medullated nerve. Proceedings of the Royal Society B, 119, 440-453.

Hodgkin, A. L. (1964). The Conduction of the Nervous Impulse, p. 66. Liverpool University Press: Liverpool.

Kugelberg, E. (1944). Accommodation in human nerves and its significance for the symptoms in circulatory disturbances and tetany. Acta Physiologica Scandinavica, 8, Suppl. 24.

Marsden, C. D., Meadows, J. C., and Merton, P. A. (1971). Isolated single motor units in human muscle and their rate of discharge during maximal voluntary effort. Journal of Physiology, 217, 12-13P.

McLeod, J. G. (1966). Digital nerve conduction in the carpal tunnel syndrome after mechanical stimulation of the finger. Journal of Neurology, Neurosurgery, and Psychiatry, 29, 12-22.
McLeod, J. G., and Wray, S. H. (1967). Conduction velocity and fibre diameter of the median and ulnar nerves of the baboon. Journal of Neurology, Neurosurgery, and Psychiatry, 30, 240-247.

Paillard, J. (1955). Réflexes et Régulations d'Origine Proprioceptive chez l'Homme. Arnette: Paris.

Sears, T. A. (1959). Action potentials evoked in digital nerves by stimulation of mechanoreceptors in the human finger. Journal of Physiology, 148, 30-31P.

Skoglund, C. R. (1942). The response to linearly increasing currents in mammalian motor and sensory nerves. Acta Physiologica Scandinavica, 4, Suppl. 12.

Veale, J. L., Rees, Sandra, and Mark, R. F. (1972). Renshaw cell activity in normal and spastic man. In New Developments in Electromyography and Clinical Neurophysiology, Vol. 3: Human Reflexes, pp. 250-261. Edited by J. E. Desmedt. Karger: Basel. 